\title{
A Effet d'un programme proposé à la lueur de l'approche actionnelle pour développer quelques compétences de la production écrite en FLE chez les apprenants de la deuxième année secondaire
}

\author{
Hussein Taha Atta ${ }^{1}$, Doaa IsmaëL El- Kady ${ }^{2}$, Dina Alaa Hussein ${ }^{3}$ \\ ${ }^{1}$ Faculté de Pédagogie, Université de Sohag \\ ${ }^{2}$ Faculté de Pédagogie, Université d'Assiout \\ ${ }^{3} X X X X X$
}

\section{Introduction}

La langue française est une des langues vivantes, les plus courantes. Pour cette raison, il est devenu nécessaire de 1 'enseigner et de l'apprendre pour permettre à l'apprenant de communiquer, d'interagir aisément et de façon vivante avec les différentes cultures et civilisations, de s'intégrer avec ce monde, de s'ouvrir aux autres, de suivre le rythme de l'urbanisation, de développer ses perceptions et d'enrichir ses expériences.

Selon Tardieu (2006), 1'enseignement des langues étrangères est passé par plusieurs évolutions: Son évolution s'est faite à partir d'une conception centrée sur le mot, puis sur la structure, et sur l'intention de communication dans 1'acte social. En somme, communiquer n'est plus seulement agir sur le monde mais agir dans le monde avec autrui.

L'approche actionnelle est apparue et est définie par le cadre européen commun de référence en traitant ses réflexions dans le processus de l'enseignement/ apprentissage des langues.

Elle est, comme la signale Elise ( 2012) : le fruit des travaux menés par des linguistes, des spécialistes de l'enseignement et des chercheurs.Elle est prônée par le CECR et les programmes actuellement en vigueur. Elle mérite par conséquent une attention toute particulière.

Selon le CECR (2001),La perspective privilégiée est, très généralement aussi, de type actionnel en ce qu'elle considère avant tout l'usager et l'apprenant d'une langue comme des acteurs sociaux ayant à accomplir des tâches (qui ne sont pas seulement langagières) dans des circonstances et un environnement donné, à l'intérieur d'un domaine d'action particulier.

Plusieurs recherches ont mis l'accent sur l'approche actionnelle en essayant d'illustrer son rôle positif et efficace dans le processus de l'enseignement / apprentissage des 
langues. À titre d'exemple, Catroux (2006), Pütz (2009), Orvoën (2012), Elise (2012) ont donné des interprétations autour de 1'approche actionnelle qui invite les apprenants à être actifs et acteurs en leur propre apprentissage, et montrent qu'il aide les apprenants à prendre la résponsabilité de leur construction cognitive, et laisse leur imagination se mettre au service de leur apprentissage.

De sa part, Shaïri (2009) a indiqué que la tâche dans une approche actionnelle, occupe une place importante dans la conception des activités d'apprentissage. Elle met les apprenants en situation d'apprentissage et de communication en les aidant à développer des strategies diverses et à augmenter leur motivation.

Nissen (2003), Ranst (2006), Sarah (2008),Puren $\quad$ (2009;2010;2011), Rosen (2010), Bazelaire (2012), ont montré 1'effet positif et efficace qu'une approche actionnelle peut produire dans l'apprentissage d'une langue étrangère. Ils ont confirmé que cette approche peut faire de la classe un espace réel et authentique de communication en français.

Selon l'approche actionnelle, l'apprenant est un acteur social confiant qui agit, s'intègre, s'implique, et participe activement et positivement aux conditions réelles en réalisant plusieurs tâches inspirées de la vie réelle. D'après ce regard, la langue n'est pas considérée comme l'objet de l'apprentissage, mais comme un outil d'accomplir des actions et des tâches, et de répondre aux exigences authentiques de la vie réelle.

L'approche actionnelle adopte, ainsi, un nouvel objectif qui vise à rendre les apprenants à être des utilisateurs actifs de la langue étrangère de façon à ce qu'ils puissent s'intégrer facilement dans les communautés natives.

En d'autres mots, Cette approche prend en considération la dimension sociale de la langue et ainsi la possibilité de s'intégrer dans une communauté européenne.

Comme le souligne Puren (2006),cette perspective actionnelle correspond à la prise en compte d'un nouvel objectif social lié aux progrès de l'intégration européenne ,celui de préparer les apprenants non seulement à vivre mais aussi à travailler, dans leur propre pays ou dans un pays étranger , avec des natifs de différentes langues- cultures étrangères. Il ne s'agit plus seulement de communiquer avec l'autre, mais d'agir avec lui en langue étrangère.

Pour cette raison, les éducateurs ont commencé à s'interesser à l'acquisition de la langue étrangère comme une habitude 
sociale et à son usage fonctionnel de la part des apprenants au climat social réel en transformant les circonstances scolaires pour être proche de la vie réelle.

L'apprentissage d'après cette approche est âxé sur la tâche qui est toute activité mettant l'apprenant en action. Elle prend la forme d'un problème ou d'une question signifiant. Elle doit être en rapport direct avec la réalité des apprenants de FLE.

Dans la pratique de la tâche, l'accent est placé sur le sens. Le CECR (2001) a donné une définition de la tâche: "Elle est un ensemble d'action finalisées dans un certain domaine avec un but défini et un produit particulier. ".

Ces tâches doivent être variées pour développer une multiplicité de compétences et d'objectifs d'apprentissage. Pour accomplir une tâche , l'apprenant recourt à ses compétences linguistiques, sociolinguistiques, et pragmatiques, et il doit prendre plusieurs décisions, et organiser son travail avec ses camarades afin de parvenir aux buts cibles.

Pour devenir un acteur social, l'apprenant doit agir avec les autres en mettant en œuvre des outils collaboratifs afin d'atteindre des buts communs au sein d'activités et d'actions collectives.
D'ailleurs , l'approche actionnelle peut développer la responsabilité de l'apprenantet en même temps l'aptitude à travailler en équipe. Cela veut dire, chaque apprenant est autonome dans son apprentissage, il doit dépendre de lui-même, de son choix et prendre un rôle qui correspond à ses capacités, ses aptitudes et ses habilités, en même temps les apprenants doivent travailler ensemble en groups dans une atmosphère de coopération et de liberté en échangeant les opinions,les informations,les expériences,et les points de vue pour atteindre le résultat identifiable.

Comme 1'explique Coste (2009): " On n'apprend pas seul, mais avec et grâce aux autres et transformant de manière personnelle et créative ,ce qui a déjà été appris par une communauté humaine"

Cette approche peut conduire à son tour à développer chez l'apprenantles compétences générales, et les compétences communicatives langagières qui sont nécessaires pour maîtriser la langue étrangère.

Selon le CECR (2001), la compétence à communiquer langagièrement $\mathrm{du}$ sujet apprenant et communiquant est mise en œuvre dans la réalisation d'activités langagières variées pouvant rélever de la réception, de la production,de l'interaction, 
de la mediation, chacune de ces modes d'activités étant susceptible de s'accomplir soit à l'oral,soit à l'écrit, soit à l'oral et à l'écrit.

Dans la présente recherche, l'accent est mis sur la production écrite. Elle est un acte de communication portant sur un système de signes graphiques que l'apprenant doit utiliser correctement en respectant les règles de fonctionnement de ces signes, pour produire des phrases significatives en formule adéquate.

La production écrite, comme toute activité rédactionnelle, est une procédure qui se compose d'un ensemble d'étapes de la préécriture, de l'écriture, et de la postécriture.Elle donne aux apprenants la liberté d'expression de soi.

Terras (2012) définit la production écrite comme, un acte signifiant qui amène l'apprenant à former et à exprimer ses idées, ses sentiments, ses intérêts, ses préoccupations, pour les communiquer à d'autres. Cette forme de communication exige la mise en oeuvre des habiletés et des stratégies que l'enfant sera appelé à maîtriser graduellement au cours de ses apprentissages scolaires.

Certes, en appliquant l'approche actionnelle, la recherche actuelle essaie de développer quelques compétences de la production écrite en FLE chez les appranants du cycle secondaire, en donnant aux apprenants la chance de rédiger librement.

\section{Problématique de la recherché}

En raison de l'importance du processus de l'apprentissage des langues étrangères et de son rôle positif dans la préparation de l'individu pour faire face à l'évolution cognitive rapide et au développement technologique, il faut s'intéresser à ce processus et jetter la lumière sur les problèmes les plus importants qui entravent l'acquisition des langues étrangères en vue d'y faire face.

Une de plus grandes contraintes dans le processus de l'enseignement/ apprentissage de la langue française, réside dans la production écrite .La compétence de la production écrite a une grande importance à la maîtrise de la langue française, Axelord et Cooper (1988)" propose leurs idées comportant, en fait, quatre points essentiels, ce que la production écrite contribue: a) particulièrement à notre façon de réfléchir, b) à notre façon d'apprentissage, c) à notre développement personnel, et d) elle nous aider, enfin, à communiquer avec d'autres personnes".

À la lumière d'études antérieures et des recherches; les didacticiens ont trouvé que la 
plupart d'apprenants souffrent de difficultés dans la production écrite en raison de l'incapacité de maîtriser ce compétence.

Les études antérieures dans le domaine de la didactique de la langue française ont mis en relief qu' il ya une faiblesse dans les compétences de la production écrite chez les apprenants qui étudient le français comme deuxième langue étrangère. Elles ont aussi montré la nécessité et l'importance du développement de ces compétences chez les apprenants ;parmi de ces études: les études de Gad (1990), Abbas (1991), El Ahwal (1991), Salem (1992), Saleh (1994), Ibrahim (1995), El Bastawssi (1996), Abdel Fattah (1997), El Battrawy (1997), Sabri (1999), Hafez (2001) , Abbas (2002), El Kassas (2002), Moussa (2002), Abd Rabou (2003), Mohammed (2003), Ezz El Arab (2003), Zekri (2003), El Kouch (2004), Farouk(2004) ， Hamed (2004), Mahmoud (2005), Mohammed (2005), SadeK (2005), Eid (2006), Abd Rabou (2008), Khalifa (2009), Agami (2010), Saleh (2015), El Naghy (2016), Mohammed (2017).

Ces études ont proposé des méthodes, des techniques d'apprentissage et des approches variées pour résoudre les problèmes reliés à la maîtrise de compétences rédactionnelles. Certains comme Ibrahim(1995) a présenté un programme proposé pour développer les compétence de la performance écrite en français chez les élèves du cycle secondaire. D'autres comme Hafez (2001) a suggéré 1' apprentissage coopératif pour but de développer des compétences de la performance écrite en français chez les étudiants du cycle secondaire. Alors que El Kouche ( 2004) a employé l'approche par le processus afin de développer des habilités d'écriture et les attitudes des étudiants du département de langue française. En outre, Mahmoud (2005) a utilisé l'approche naturelle visant à développer l'expression écrite en langue française chez les étudiants du cycle préparatoire. De sa part, Abd Rabou(2008) a voulu développer des compétences de la lecture, de l'écriture,et de l'autonomie chez les étudiants du département de français à la faculté de pédagogie au cours d'un programme proposé basé sur l'auto - apprentissage. D'autres comme Khalifa ( 2009) a élaboré un modèle constructiviste ayant pour but de développer les compétences de 1'expression écrite chez les élèves du cycle secondaire pour apprendre la grammaire de français. Et comme Agami (2010) a proposé un programme basé sur l'approche du processus de l'écriture pour développer quelques compétences de la production écrite et les attitudes des élèves du cycle secondaire. Alors que Saleh (2015) a 
présenté une unité proposée basé sur l'approche actionnelle comme alternative à la méthode traditionnellepour but de développer quelques compétences de la compréhension écrite chez les étudiants à la faculté de pédagogie.

Malgré ces grands efforts dans ce domaine, les apprenants qui étudient la langue française au cycle secondaire souffrent et rencontrent beaucoup de difficultés aux activités de la production écrite, cela affecte à son tour négativement la maîtrise de la langue française entièrement.

La chercheuse a effectué une étude pilote visant à évaluer les compétences de la production écrite chez les élèves de la deuxième année du cycle secondaire.Elle a eu recours à un test de production écrite (préparé par la chercheuse), et elle l'a appliqué sur 30 élèves. D'après l'analyse des réponses de cet échantillon, la chercheuse a trouvé que:

$85,7 \%$ d'apprenants ne peuvent pas rédiger quelques phrases complètes et significatives sous certaines images données.

$$
48,6 \% \text { d'apprenants ne sont pas }
$$
capables de construire des phrases simples et complètes à l'aide de quelques mots

$71,4 \%$ d'apprenants ne peuvent pas traduire correctement leurs idées en langue française pour rédiger un texte significatif.
$88,6 \%$ d'apprenants ne peuvent pas mettre les phrases en ordre chrolonogique pour formuler des textes complets et compréhensibles.

$51,4 \%$ d'apprenants ne sont pas capables de respecter le style et la mise en page.

D'après ces constats, la chercheuse a essayé dans la présente recherche, de mettre en œuvre des activités rédactionnelles en fonction d'un scénario d'apprentissage conçu selon la perspective actionnelle.

\section{Question de la recherché}

Cette recherche tente de répondre à la question principale suivante:

"Quelle est l'effet d'un programme proposé à la lueur de l'approche actionnelle pour développer quelques compétences de la production écrite en FLE chez les élèves du cycle secondaire?"

\section{Objectif de la recherché}

Cette recherche a pour objectif de vérifier l'effet d'un programme proposé à la lueur de l'approche actionnelle pour développer quelques compétences de la production écrite en FLE chez les élèves du cycle secondaire.

\section{Importance de la recherché}


L'importance de cette recherche se manifeste sur deux plans:

\section{- Sur le plan théorique}

a. Le cadre théorique de la recherche actuelle peut présenter un cadrage théorique cohérent et exhaustif sur l'approche actionnelle et la production écrite.

b. Une liste de quelques compétences de la production écrite pour les apprenants du cycle secondaire. Cette liste peut-être considérée comme référence pour beaucoup d'autres recherches dans ce domaine.

c. Un scénario d'apprentissage conçu selon l'approche actionnelle et qui peut être utilisé dans un contexte d'apprentissage de la langue française écrite.

\section{- Sur le plan pratique}

a. Présenter une liste de quelques compétences de la production écrite.

b. Élaborer un programme basé sur l'approche actionnelle pour développer quelques compétences de la production écrite.

c. Élaborer un guide de l'enseignant selon l'approche actionnelle.

d. Élaborer un test pour évaluer les compétences de la production écrite.

\section{Limites de la recherche}

Cette recherche se limite à:

a- Un échantillon des apprenants de la deuxième année du cycle secondaire (30 élèves)

b- Développer quelques compétences de la production écrite

- Compétences de fond et de forme

- Compétences grammaticales

- Compétences de contenu, de syntaxe

- Compétences de jugement, de style, d'organisation, et de vocabulaire.

\section{Méthodologie}

La présente recherche a utilisé la méthode (quasi - expérimentale) en adoptant un seul groupe (groupe expérimental) et en appliquant des instruments de la recherche pour vérifier l'effet du programme proposé au développement de quelques compétences de la production écrite.

\section{Outils de la recherche}

On a élaboré les instruments suivants:

a- Une liste de quelques compétences de la production écrite.

b- Un programme fondé sur l'approche actionnelle en vue de développer quelques compétences de la production écrite.

c- Un guide de l'enseignant selon 1'approche actionnelle. 
d- Un pré - post test pour évaluer les compétences de la production écrite.

\section{Terminologies}

\section{L'approche actionnelle}

Pour Barrié (2012),l'approche actionnelle prépare l'apprenant à utiliser la langue dans des situations imprévues (comme dans la vie courante où l'on ne connait pas à l'avance le contenu du dialogue). On donne alors à l'apprenant les armes pour communiquer et agir dans n'importe quelle situation.

Comme le souligne C.Puren (2013), la perspective $[. .$.$] de type actionnel considère$ avant tout l'usager et l'apprenant d'une langue comme des acteurs sociaux ayant à accomplir des tâches (qui ne sont pas seulement langagières) dans des circonstances et un environnement donnés dans un domaine particulier.

Et pour cette recherche: signifie qu'un programme vise à développer les compétences de la production écrite des textes en (FLE), en présentant aux apprenants un ensemble de tâches et d'activités rédactionnelles qui doivent être réalisées à la lumière d'un scénario proposé.

\section{La production écrite}

Claudette cornaire et Patricia Mary Raymond, dans leur ouvrage (1999)" la production écrite" dissent que ,écrire c'est conduire les étudiants à produire des écrits diversifiés , récits, textes explicatifs, règles de jeux, textes argumentatifs, textes poétiques. De même elles définissent la production écrite disant que : " la production écrite n'est pas la simple transposition de quelques connaissances, mais une construction complexe qui résulte de l'interaction entre le scripteur, le texte et le context ou encore la situation d'interlocution.

Dans la théorie de la communication, la production écrite est: "une activité de production d'un texte écrit vue comme une intégration entre une situation d'interlocution et un scripteur dont le but est d'énoncer un message dans un discours écrit" (Robert, 2002).

Et pour cette recherche: $C^{\prime}$ est le fait de disposer correctement les codes linguistiques pour produire par écrit des textes authentiques en racontant des actions et des événements réels inspirés de la vie quotidienne et en respectant les règles de la langue écrite.

\section{Procédures de la recherche}

Pour répondre à la question de cette recherche, la chercheuse a suivi les démarches suivantes:

a. Déterminer les compétences de la production écrite pour les élèves de la 
deuxième année du cycle secondaire; et ceci au cours de:

- Étudier- consulter des ouvrages et des études antérieurs concernant ce domaine.

- Élaborer une liste de quelques compétences de la production écrite.

- Exposer cette liste à un jury de spécialistes

- Modifier la forme de cette liste d'après les points de vue des membres du jury.

b. Elaborer un programme à la lueur de l'approche actionnelle pour développer quelques compétences de la production écrite; et ceci au cours de:

- Élaborer un programme proposé (ses objectifs - son contenu basé sur l'approche actionnelle $\quad-\quad$ son évaluation)

- Elaborer des instruments de mesure nécessaires à l'évaluation des compétences de la production écrite (un pré-post test).

- Exposer ces instruments à un jury de spécialistes

- Modifier les formes de ces instruments d'après les points de vue des membres du jury.
- Choisir au hasard un échantillon de (30 élèves) parmi les apprenants de la deuxième année du cycle secondaire.

- Appliquer le pré- test sur le groupe expérimental avant l'application du programme.

- Appliquer le programme proposé sur le groupe expérimental.

- Appliquer le post test sur le groupe expérimental après l'application du programme.

- Analyser statistiquement les résultats obtenus.

- Interpréter les résultats.

- Présenter des recommandations et des suggestions de l'étude.

\section{Cadre théorique}

\section{Axe (1): L'approche actionnelle}

L'approche actionnelle modélée en 2001 par les travaux du Conseil de 1'Europe qui a élaboré le Cadre européen commun de référence pour les langues, représente une nouvelle orientation au domaine d'enseignement - apprentissage des langues étrangères.

Trim (1997) souligne que, cette orientation est la marque des travaux du Conseil de l'Europe depuis le début des années 70; elle considère 1'apprentissage des langues comme une préparation à une 
utilisation active de la langue pour communiquer. «L'apprentissage fondé sur la tâche » est, tout naturellement, une tendance forte et croissante dans le cadre de l'approche communicative.

La présente recherche jette la lumière sur l'approche actionnelle désormais (PA) comme l'une des approches principales pour apprendre le français langue étrangère (FLE) et qui peut contribuer de façon tangible à faciliter $l^{\prime}$ apprentissage et $l^{\prime}$ usage correcte et authentique de cette langue en différentes situations hors de classe.

Le CECRL (2001) pose les bases théoriques de l'approche actionnelle, et il en propose une définition, la perspective privilégiée ici est, très généralement aussi, de type actionnel en ce qu'elle considère avant tout l'usager et l'apprenant d'une langue comme des acteurs sociaux ayant à accomplir des tâches (qui ne sont pas seulement langagières ) dans des circonstances et un environnement donnés, à l'intérieur d'un domaine d'action particulier. Si les actes de parole de réaliser dans des activités langagières, celles-ci s'inscrivent ellesmêmes à l'intérieur d'actions en contexte social qui seules leur donnent leur pleine signification.

D'après Sarah (2008), cette approche actionnelle développe l'idée essentielle que la langue est enseignée et apprise pour et par l'action sociale. De plus, elle favorise la formation des citoyens de demain nécessiteux de communiquer avec des personnes ne parlant pas la même langue, en pointant du doigt que dans le monde extrascolaire, la communication n'est pas une fin en soi, mais un moyen au service d'activités socialement significatives.

Comme le dit Shaïri (2009, 43), la perspective actionnelle est une nouvelle approche didactique, qui offre un nouveau sens à l'interaction entre les apprenants dans le cadre de l'apprentissage et au sujet de la réalisation de la tâche.

Comme le propose Rosen (2009), l'apprentissage, dans la perspective actionnelle, est conçu comme une activité sociale; la langue cible est développée en interagissant et en recevant les apports de l'entourage.

De sa part, Bourguignon (2010) constate que, dans la perspective actionnelle, l'apprenant-usager de la langue peut être considéré comme un ingénieur qui est confronté à une situation et qui doit définir les connaissances (avec l'aide de l'enseignant bien évidemment, $[\ldots]$ )dont il a besoin pour résoudre ce problème

Pour Barrié (2012): l'approche actionnelle prépare l'apprenant à utiliser la 
langue dans des situations imprévues (comme dans la vie courante où l'on ne connait pas à l'avance le contenu du dialogue). On donne alors à l'apprenant les armes pour communiquer et agir dans n'importe quelle situation.

Gomes (2012) souligne que, la perspective actionnelle se fonde sur la valeur didactique des situations d'immersion totale en langue étrangère, où le sujet-apprenant va être confronté à des nouvelles expériences socio- linguistiques et par ce biais (apprendre sur le tas), c'est-à-dire, apprendre sur le déroulement de la tâche à effectuer, car l'action s'impose au quotidian.

\section{À la lumière d'interprétations précédentes, on peut constater que:}

1- L'approche actionnelle est une révolution didactique, qui recourt à la communication, à l'interaction et à la coaction.

2- L'approche actionnelle adopte de nouveaux concepts: 1'action, l'agir , l'engagement.

3- D'après cette approche, l'apprenant est un acteur social confiant qui agit, s'intègre, s'implique et participe activement et positivement aux conditions réelles réalisant plusieurs tâches proches de la vie réelle. En conformité avec cette approche, on peut dire que l'apprenant devient un usager de la langue française comme $1^{\prime}$ usager natif de cette langue ( les français).

4- L'approche actionnelle prépare les apprenants pour faire face aux différents défis quand ils utilisent la langue cible hors de la classe.

5- À la lueur de l'approche actionnelle, les actes ne sont pas seulement de parole et ils doivent se produire hors du domaine purement langagier, cela veut dire ils n'ont de sens que par rapport aux actions sociales.

6- La contextualisation est une partie intégrante du processus d'apprentissage de la langue étrangère à la lumière de l'approche actionnelle, parce que l'apprentissage de cette langue ne doit pas être decontextualisé, mais il doit être en différents contextes situationnels, en demandant d'apprenants de faire un nombre d'actions qui ont du sens pour eux.

7- Tous les actes de communication doivent avoir de sens pour l'apprenant afin de ressentir le besoin de s'impliquer et de s' engager dans les situations de communication. Alors, $l^{\prime}$ accent est mis sur le sens plus que la forme.

8- L'apprenant comme un acteur social doit agir dans les grands domaines de sa vie 
sociale (éducationnel, personnel, professionnel, public). À chacun d'eux, il doit faire face aux différents contextes. Ces contextes déterminent un certain nombre de situations de communication. Ces situations forment les tâches demandées à accomplir.

\section{Les objectifs de l'approche actionnelle:}

L'objectif de l'approche actionnelle est de permettre aux apprenants d'apprendre la langue étrangère et de l'utiliser socialement pour interagir, s'intégrer et travailler efficacement et avec confiance avec d'autres locuteurs natifs au monde extérieur.

En d'autres termes, cette nouvelle approche a pour but d'intégrer la co-action sociale à l'apprentissage de la langueétrangère et de rendre apprenant comme un citoyen qui établit des relations sociales avec des gens de langues et de cultures différentes.

Gonzalez (2012) affirme la même idée en indiquant que, l'objectif visé est la co-action sociale, c'est-à-dire l'action finalisée et conjointe par le biais de l'apprentissage d'une langue dans un cadre social donné, (...). Dans le monde actuel, l'acteur social qu'est l'apprenant n'est plus une personne qui cohabite seulement avec des étrangers,mais qui agit en société, c'est-à-dire conjointement avec d'autres. C'est alors en agissant et en travaillant ensemble que se construisent des représentations communes.

Rosen (2009) confirme que le but de cette approche est de former des personnes capables de s'intégrer dans les pays d'Europe pour une durée assez longue afin qu'elles puissent y effectuer une partie de leurs études ou une partie de leur carrière.

\section{La tâche comme axe principale dans la perspective actionnelle:}

L'approche actionnelle est centrée sur la notion de la tâche. Alors, il est nécessaire d'expliquer le terme de tâche, mais les définitions du terme " tâche" varient d'après les chercheurs et leurs points de vue.

La tâche est un élément clé dans l'approche actionnelleet son accomplissement peut permettre aux apprenantsde développer facilement leurs capacités et leurs différentes compétences.

Nunan (1991) considère que " la conception d'une tâche requiert la prise en compte de six paramètres: les objectifs, les données de départ, les activités que 1' apprenant sera amené à réaliser, les rôles respectifs de l'enseignant et des apprenants, et enfin le dispositif".

La notion de tâche selon le CECR (2001): "est définie comme tâche toute visée 
actionnelle que l'acteur se représente comme devant parvenir à un résultat donné en fonction d'un problème à résoudre, d'une obligation à remplir, d'un but qu'on s'est fixé".

Pour Coste ( 2009), la notion de tâche renvoie à une action finalisée, avec un début, un achèvement visé, des conditions d' effectuation, des résultats constatables, à la différence de l'exercice, qui est essentiellement langagier ponctuel et répétitif, la tâche n'est pas seulement langagière ; elle peut également mobiliser plusieurs compétences et stratégies diversifiées.

D'après puren (2013), une tâche est une activité que les apprenants doivent réaliser consciemment au sein d'un dispositif donné avec une visée qui peut être orientée langue, processus, procédure, communication, résultat ou produit.

Simplement, la tâche est l'axe principale dans l'approche actionnelle. Elle est un acte qui a un objectif, un contenu et un plan déterminé. Elle vise à faciliter l'apprentissage de la langue étrangère en passant d'activités simplifiées aux activités les plus complexes.

Les tâches doivent être variées, proches de la vie réelle et complexes en fournissant plusieurs défis devant les apprenants qui doivent utiliser efficacement la langue pour accomplir ces tâches. Cela peut donner à l'apprenant la possibilité de communiquer, d'agir et de s'intégrer dans n'importe quelle situation de la vie quotidienne.

\section{Le scénario d'apprentissage selon le}

\section{CECR:}

Le scénario est la phase la plus importante pour accomplir la tâche finale.Il signifie comme un design clair et facile à comprendre qui décrit les démarches et les procédures séquentielle doivent être mises en œuvre durant l'accomplissement des tâches. Il peut être aussi considéré comme un plan qui a été accepté par le groupe du travail.

Puren (2013) définit la scénario comme un ensemble prédéfini d'actions (en société ou en simulation) ou de tâches "scénario pédagogique" articulées dans la même chronologie.

Selon Nissen (2004), le scénario peut ici être défini comme étant la mise en scène de la réalisation de la tâche en langue étrangère dans le dispositif pédagogique. Le scénario correspond à un parcours dans le dispositif proposé et qui contient des aides et ressources de différentes sortes.

D'après Springer (2009, 520), scénariser signifie concevoir un environnement finalisé en termes de rôles, d'actions, de ressources, 
de méthodes, de régulation /médiation, de résultat.

Dans la présente recherche, pour élaborer les tâches rédactionnelles au programme proposé, on a adopté le scénario de Bourguignon.

\section{Le scénario d'apprentissage - action}

Tenant compte des orientations du Cadre, Bourguignon (2007) a défini le scénario d'apprentissage-action comme, une simulation basée sur une série de tâches communicatives, toutes reliées les unes aux autres, visant l'accomplissement d'une mission plus ou moins complexe par rapport à un objectif. Cette série d'activités amène à la réalisation de la tâche finale.

De sa part, Bourguignon (2007) ajoute que, En préparant un scénario $\mathrm{d}^{\prime}$ apprentissage-action, ce n'est pas tant le support qui doit faire l'objet d'une analyse approfondie, que la mission qui est demandée, car c'est elle qui va guider de manière intégrée les tâches communicatives demandées aux élèves en relation avec les activités de communication langagière qui seront les unes après les autres au service de l' accomplissement de la tâche finale .

En définitive, Le scénario adopté consiste à concevoir des séquences structurées autour d'un ensemble de tâches finales complexes qui doivent être confiées aux apprenants afin de les accomplir. Pour réaliser la tâche finale, les apprenants doivent passer par différentes tâches intermédiaires. Mais ils ne possèdent pas toutes les connaissances ou savoir-faire nécessaires pour réaliser ces tâches.

Par conséquent, les tâches exigent la mise en pratique plus ou moins de différentes activités langagières permettant aux apprenants de recevoir, d'acquérir et de développer les savoirs, les capacités et les compétences.

On a mentionné plus tôt que la tâche est un groupe d'activités. Ces activités comprennent les activités de réception, de production, d'interaction, d'expression et de compréhension qui aident l'apprenant à explorer, à comprendre, à observer, à penser, à exprimer, à critiquer, à analyser, à construireet à produire.

Certaines activités peuvent être courtes que d'autres, mais elles sont très importantes et utiles pour l'accomplissement de la tâche finale.

Pour Willis (2009), il est important que les apprenants travaillent librement dans ces activités de réemploi. Elles doivent leur donner l'occasion de réutiliser le langage appris, mais si elles sont précédées immédiatement par un travail sur la forme, 
elles ne seront pas traitées comme des activités sur le sens mais comme une invitation il montrer la manière dont on contrôle les formes.

Enfin, ces activités peuvent donner à l'apprenant l'occasion d'être un véritable acteur dans la société de la classe. Et elles peuvent l'encourager d'écrire activement en langue étrangère.

\section{La structure d'une unité actionnelle}

On a mentionné déjà que la tâche est une série d'activités ou d'actions significatives et finalisées que les apprenants doivent accomplir pour acquérir les nouvelles connaissances et développer les différentes compétences. Alors, la tâche bien préparée permet aux apprenants de parvenir aux buts didactiques visés.

En d'autres mots, La mise en pratique de l'approche actionnelle exige de concevoir des unités didactiques fondées sur l'articulation de tâches actionnelles significatives à accomplir, en éveillant l'intérêt chez les apprenants à maîtriser la langue cible de manière authentique.

Selon son point de vue, Orvoën (2012), Pendant la phase de préparation, les apprenants vont être déjà confrontés et sensibilisés aux éléments lexicaux et grammaticaux les plus importants et nécessaires. Puis c'est lors de la préparation et de la planification que l'on pourra porter son attention sur la forme afin d'exprimer ce que l'on a à dire ou à faire et d'améliorer son travail. On peut laisser les apprenants réaliser la préparation sans étude formelle afin de travailler la compétence d'interaction souvent spontanée. Puis le regard apporté à la forme lors de la planification aidera l'apprenant à le rassurer, à lui donner les connaissances utiles pour pouvoir réaliser la tâche finale.

D'après Tano (2010), les élèves doivent avoir une raison valable pour utiliser la langue et éviter toute pratique mécanique et décontextualisée. Si savoir communiquer consiste à savoir agir socialement, il faut donc proposer à l'élève d'agir avec les autres en utilisant la langue. En faisant des activités contextualisées, l'élève découvre, par luimême, dans son interaction avec ses camarades, les règles de fonctionnement de la langue. Le professeur s'assure ainsi d'encrer la production langagière à partir de l'identité de l'élève (son expérience, son point de vue).

\section{Les nouveaux rôles de l'enseignant}

À la lueur de l'approche actionnelle, l'enseignant assume de nouveaux rôles. Il devient un guide qui dirige les apprenants, incite leur motivation, facilite leur apprentissage, leur offre les instructions et les 
directives appropriées, les encourage de faire le meilleur, intervient pour les aider à résoudre les problèmes rencontrés, leur offre les conseils, facilite les interactions entre eux, prend en considération leurs besoins et les différences individuelles entre eux , les aide à accomplir les tâches confiéeset enfin les évalue équitablement et leur fournit des feedbacks nécessaires pour parvenir à l'objectif final.

De sa part, Peris (2009), «enseigner, ce n'est pas seulement fournir des contenus et aider les élèves à les apprendre, mais les aider à apprendre à apprendre ».

Shaïri (2009) constate que, la perspective actionnelle entraine également une redéfinition du rôle de l'enseignant(e). Il/Elle doit désormais orienter les apprenants vers les démarches à suivre, intervenir pour les conseiller, leur donner confiance et les inciter à la communication et non pas chercher à leur transmettre son savoir magistral.

\section{Les nouveaux rôles de l'apprenant}

L'apprenant devient un acteur social responsable qui prend un grand nombre de rôles. Il participe à planifier, à organiser les tâches à accomplir, choisit un rôle s'accorde avec ses désirs, ses aptitudes, ses habiletés, ses besoins et ses énergies, interagit, s'implique activement aux diverses activités, collabore avec ses camardes, réflechit, analyse, pense, s'évalue ( son propre produit), évalue les autres apprenants et fait face aux diverses contraintes avec confiance afin d'atteindre le but identifiable.

Pour Toruç (2013), l'apprenant n'est pas seulement récepteur de connaissances, de savoirs mais un réalisateur qui les met en œuvre; c'est-à-dire, un acteur qui est capable de montrer ses compétences, ses savoir faire.

Selon Puren (2009), afin de mettre en œuvre les tâches à accomplir, les apprenants doivent:

1. Prendre conscience de l'existence d'un besoin ou problème dont la solution nécessite de l'information.

2. Savoir identifier et définir avec précision l'information nécessaire pour satisfaire le besoin ou résoudre le problème.

3. Savoir déterminer si l'information nécessaire existe ou non, et, dans la négative

4. Savoir trouver l'information nécessaire quand on sait qu'elle existe

5. Savoir créer, ou faire créer, l'information qui n'est pas disponible (créer de nouvelles connaissances)

6. Savoir bien comprendre l'information trouvée, ou à qui faire appel pour cela, si besoin est. 
7. Savoir organiser, analyser, interpréter et évaluer l'information, y compris la fiabilité des sources.

8. Savoir communiquer et présenter l'information à autrui sur des formats/ supports appropriés/ utilisables.

9. Savoir utiliser l'information pour résoudre un problème, prendre une décision, satisfaire un besoin.

10. Savoir préserver, stocker, réutiliser, enregistrer et archiver l'information pour une utilisation future.

11. Savoir se défaire de l'information qui n'est plus nécessaire et préserver celle qui doit être protégée.

\section{Axe (2): La production écrite en classe de français langue étrangère (FLE)}

Une des exigences de l'apprenant est d'utiliser la langue écrite pour interagir avec les autres et s'exprimer aisément. La production écrite est un outil de communication qui prend le rôle de répondre àcette exigence et traduit sa pensée et son expression de soi.

Cela veut dire que la production écrite est un des moyens de communication entre les êtres humainset elle permet à l'apprenant de traduire ses idées et de mieux définir et cristalliser ses points de vue et ses opinions dans un contexte linguistique caractérisé par la richesse du vocabulaire, des phrases complètes et l'intégrité de structures et d'expressions.

D'après Charline (2010), la production écrite est un élément essentiel de l'apprentissage de la langue écrite.

Actuellement, les chercheurs accorde une attention particulière à la production écrite dans leurs recherches, leurs études récentes et aussi dans les conférences et les seminaires qui traitent le processus d'enseignementapprentissage des langues étrangères.

La production écrite n'est pas une activité facile. Elle est acquise à travers de l'étude, de l'apprentissage, de l'entraînement et de l'expérience. Elle exige aussi un effort mental considérableet une capacité graphique et intellectuelle.

Comme le souligne Sinh (2006), rédiger un texte en langue étrangère, ce n'est pas seulement écrire de belles phrases, c'est aussi savoir comment les phrases sont uiilisées à des fins de communication.

Abdelli (2011) assure sur la même idée, il est indéniable que rédiger est un processus très complexe, et il n'est pas facile de faire acquérir aux élèves une compétence en production écrite, car l'écriture ne consiste pas à produire un ensemble de structure linguistique correspondant à une suite de 
phrases bien exprimées, mais c'est surtout concrétiser une série de procédures de résolution de problèmes qu'il n'est pas facile de distinguer et structure.

Darmawangsa (2008) affirme que la compétence de la production écrite est l'une la plus difficile à adapter par rapport à d'autres compétences.

Selon Agami (2010), la didactique de la production écrite en langue étrangère a évalué ces dernières années. Elle a pris un ensemble de physionomies dans plusieurs approches pédagogiques qui ont marqué l'enseignement de l'écrit.

\section{Importance de la production écrite}

Comme le dit Sara (2011), la production écrite est une activité très importante pour mieux faire centrer l'enseignement du français langue étrangère. Elle est l'objet de recherche des travaux qui s'occupent à identifier les moyens et les méthodes les plus adéquates pour une didactique efficiente d'elle. Ces recherches ont fleuri en divers domaines; la production écrite a eu sa place dans plusieurs méthodes et approches didactiques, d'autres travaux ont donné des modèles d'écritures qui se centrent sur l'analyse des processus de la pratique de la production écrite d'une part, et l'analyse du produit fini de cette activité de l'autre part.
Ainsi elle revête une importance particulière pour les apprenants au moins pour deux raisons : d'une part, l'écriture a une fonction d'apprentissage dans les différents disciplines ; d'autre part, les pratiques d'écriture sont socialement et scolairement différenciatrices ( Plane, 2004).

Axelord et Cooper (1988) propose leurs idées comportant, en fait, quatre points essentiels, ce que la production écrite contribue: a) particulièrement à notre façon de réfléchir, b) à notre façon d'apprentissage, c) à notre développement personnel, et d) elle nous aider, enfin, à communiquer avec d'autres personnes.

La production écrite réflète les effets du développement des compétences liées aux autres trois arts (de lecture, d'écouteet de parole), en ajoutant la reflexion qui est la cinquième de ces arts.

La production écrite donne aux apprenants la possibilité de réflechir, de choisir les idées appropriées et de sélectionner du vocabulaire, des énoncés et des structures convenables pour traduire écritement leurs idées. En outre elle ouvre le domaine devant l'enseignant pour identifier les lacunes et les forces chez les apprenants et tenter de rémedier aux difficultés rencontrées. Grâce à la production écrite, l'enseignant peut aussi détecter et découvrir 
les apprenants qui possèdent des talents spéciaux et ayant des compétences et des habilétés écrites spécifiques dans le but de les encourager et de les guider.

À la lumière de cette importance et cette valeur éducative pour la production écrite, il est nécessaire d'augmenter l'intérêt à son enseignement/ apprentissage et les enseignants doivent développer les compétences rédactionnelles chez les apprenants (dès le dessin des mots correctement jusqu'à la rédaction d'un sujet complet et intégré).

\section{Objectifs de la production écrite}

L'objectif principal de la production écrite est d'améner les apprenants à saisir le sens global du texte, à comprendre son organisation et à produire des textes liés à leur réalité quotidienne en utilisant des expressions familières et quotidiennes qui visent à satisfaire des besoins concrets et en respectant les règles de la langue écrite.

Comme l'indique Agami (2010), la production écrite vise donc à réaliser la communication entre les gens afin de satisfaire leurs besoins, les domaines varient selon la nature du travail et du public visé .

Sara (2011) constate que l'enseignement de la production écrite en français langue étrangère a pour objectif de mener les apprenants à la maîtrise d'une compétence de la communication écrite. Elle ajoute que, la grammaire, l'orthographe, la conjugaison, et le vocabulaire ont pour objectif de mener l'apprenant à écrire correctement en français langue étrangère,une faute commise est susceptible de changer la structure sémantique de la phrase. D'autre part ils aident les apprenants à l'apprentissage d'une compétence en production écrite, grâce aux diversités d'exercices dans chaque activité dont les apprenants utilisent des phrases simples et complexes, prennent du temps considérable dans les phases d'applications, ils s'apprennent comment produire un écrit par le biais d'une application correcte des règles.

En résume, on peut dire que l'approche actionnelle est une orientation didactique très bénéfique au parcours d'enseignement/ apprentissage des langues étrangères. Cela veut dire que cette approche vise à préparer les apprenants aux défis auxquels ils font face quand ils utilisent la langue étrangère hors de classe. Par conséquent, ils peuvent maîtriser efficacement cette langue et devenir des usagers de la langue comme les natifs.

D'ailleurs, à la lueur de cette approche, la langue devient non seulement un instrument de communication, mais aussi un 
instrument d'action et d'agir socialement aux travaux réels.

Comme l'explique Pérès (2013) : (La perspective actionnelle vise l'acquisition des cinq compétences langagières qui sont définies dans le CECRL : compréhension orale, compréhension écrite, production orale en continu, production orale en interaction et production écrite).

Sans doute, pour se familiariser avec la langue française écrite et produire des textes authentiques liés à la vie quotidienne en respectant les codes du type d'écrit et la correction de la langue écrite, les apprenants ont besoin d'être en contact réellement avec les phrases et les textes.

La présente recherche propose un programme basé sur l'approche actionnelle visant à rémedier aux difficultés de la production écrite chez les apprenants et les rendre capables de former correctement les mots, afin de les utiliser ensuite à la formulation des phrases organisées en paragraphes, en vue de rédiger des textes compréhensibles, significatifs, acceptables et authentiques, à travers de leur présenter un certain nombre de tâches rédactionnelles en fonction d'un scénario pédagogique proposé $\mathrm{d}^{\prime}$ après cette approche.
Cela veut dire que l'approche actionnelle peut donner aux apprenants la chance de produire plusieurs textes liés aux expériences de vie, aux faits et aux événements réels qui se sont passés déjà dans leur vie personnelle.

Tout cela peut conduire à son tour à développer les compétences de la production écrite chez eux.

\section{Les instruments de la recherche et ses procédures}

1- Elaboration de la grille de compétences de la production écrite

Cette grille a visé à identifier les compétences de la production écrite compatibles aux apprenants de la deuxième année au cycle secondaire.

Après avoir élaboré la grille de compétences de la production écrite, on l'a présenté aux membres du jury.

\section{2- Elaboration du Test de la production écrite}

Objectifs du test: Le pré-test et le post test utilisés lors de cette étude ont visé à mesurer l'éffet d'un programme proposé à la lueur de l'approche actionnelle pour développer quelques compétences de la production écrite chez les apprenants de la deuxième année du cycle secondaire. 
Après avoir élaboré le test sous sa forme initiale, on l'a présenté aux membres du jury en leur demandant de déterminer si:

- Le test est au niveau des apprenants de la deuxième année secondaire.

- Le test est pertinent et compatible à mesurer les compétences de production écrite visées.

- Modifier les questions mal formées.

Application du test: On a fait passer ce test aux membres de l'échantillon, avant et après l'enseignement de l'unité proposée, afin de mesurer son efficacité.

\section{3- Elaboration du programme proposé:}

Les objectifs généraux du programme:

Il s'agit de l'objectif général, le comportement qu'on vise à réaliser après l'application du programme proposé.

- Ce programme a été conçu dans le but de permettre aux apprenants de se familiariser avec la structure du texte écrit à travers de mieux comprendre les textes proposés aux leçons, et de mieux apprendre les étapes exigées pour réaliser des tâches liées à la production écrite de textes complets, clairs et cohérents.

- En outre, ce programme a visé aussi à adopter et à appliquer les principes de l'approche actionnelle pendant la réalisation des tâches écrites qui sont confiées aux apprenants. Cela à travers de leur demander de rédiger des textes autour d'événements et des actions inspirés de leur vie quotidienne, cela veut dire qui se passent réellement à la vie.

- Tout cela, peut contribuer à son tour à développer les compétences de la production écrite, à maîtriser l'apprentissage de la langue française et à utiliser concrètement et avec aisance cette langue dans des activités sociales réelles dans et hors de classe.

- Les objectifs secondaires du programme: Il s'agit de l'objectif opérationnel, le comportement qu'on vise à atteindre après l'étude d'un cours précis, ou une unité déterminée.

En vue d'élaborer le programme proposé, la chercheuse a réfléchi sur la nature de l'apprentissage de la production écrite à l' aide de:

- Les études et les recherches antérieures qui s'intéressent à l'étude de 1 'influence de quelques stratégies et d'approches pédagogiques sur les compétences langagières des apprenants, les compétences de l'écriture, la production écrite.

- Les compétences de la production écrite en général et celles du cadre commun européen en particulier. 
- Étude les références portant sur la méthodologie de l'approche actionnelle comme: CECR (2001), Bourguignon (2007), Rosen (2009), Tano (2010), Gouton (2013), Ellis (2012)....

- Consultation des livres et des sites d'Internet concernant l'explication de la production écrite de langue en général et de la langue française en particulier comme: Charline (2010), Abdelli (2011).

Pour élaborer les tâches rédactionnelles au programme proposé, on a adopté le scénarion de Bourguignon.

\section{Contenu du programme:}

Le programme proposé a compris sept leçons avec une leçon zero.

Ces leçons ont été tiré du livre (BRAVO).

Ces leçons ont pour but de développer quelques compétences de la production écrite en appliquant l'approche actionnelle.

\section{Chaque leçon a comporté:}

- Les compétences à développer, la durée et le nombre des tâches de chaque leçon.

- Quelques consignes pour les apprenants avant de commencer les tâches de la leçon.

- Le contenu de la leçon

- L'évaluation de chaque leçon.

\section{Types d'évaluation:}

La chercheuse a préparé des outils pour mesurer l'éfficacité du programme proposé à la lueur de l'approche actionnelle.

On peut distinguer trois types d'évaluation:

\section{1- Diagnostique}

On a évalué le niveau des apprenants avant l'enseignement du programme proposé en appliquant le pré-test de la production écrite afin de déterminer le niveau des apprenants avant de commencer l'enseignement du programme.

\section{2- Formative}

Selon laquelle, on a appliqué une grille d'évaluation de compétences de production écrite comme une évaluation durant l'enseignement de chaque leçon pour évaluer le niveau de chaque groupe et faire une comparaison entre son niveau par rapport au niveau des autres groupes.

Quant à l'évaluation formative, les produits écrits des apprenants ont été évalués par la chercheuse. Chaque groupe a reçu des notes pour chaque copie écrite jusqu'à la dernière version. Cette évaluation a été basée sur les critères de la grille de compétences de la production écrite pour les apprenants de la deuxième année secondaire. 


\section{3- Sommative}

Après l'application du programme proposé, on a évalué le niveau des apprenants sommativement en appliquant le post-test de production écrite, et on a fait la comparaison entre leur niveau d'acquisition de compétences avant et après 1'enseignement du programme proposé.

Autrement dit, ce qui concerne l'évaluation sommative, elle était à travers le post-test qui a eu lieu à la fin de l'expérimentation, en visant de mesurer le développement des compétences et des sous-compétences de production écrite chez les apprenants, et de mésurer l'efficacité du programme proposé.

\section{Instruments de collecte des données}

Test de production écrite:

- Le pré test pour examiner le niveau des apprenants du groupe expérimentale en production écrite en FLE avant d'enseigner le programme proposé.

- Le post test pour examiner le niveau des apprenants du groupe expérimentale en production écrite en FLE à la fin du programme proposé.

\section{Elaboration d'un guide de maître:}

La chercheuse a organisé un guide en vue d'aider celui qui enseigne le programme proposé, ce guide a contenu le suivant:

- Les objectifs du programme
- Le contenu du programme

- Les activités proposées

- l'évaluation

- les stratégies utilisées

- les réponses attendees Après avoir préparé le programme dans sa forme initiale, on $\mathrm{l}^{\prime}$ a présenté au jury.

\section{Étapes de l' expérimentation:}

Après l'élaboration de la grille de compétences de la production écrite, le test, la fiche d'évaluation de la performance des apprenants pendant l'application du programme proposé, on a commencé l'étude expérimentale selon les étapes pédagogiques suivantes:

\section{Choix de l'échantillon de la recherche}

La chercheuse a choisi, un seul group expérimental composé de 30 apprenants de deuxième année secondaire au lycée de (khadiga Youssef pour les filles) à Assiout.

\section{Application du pré-test sur l'échantillon de la recherche:}

Avant de commencer l'enseignement de l'unitée proposée, la chercheuse a appliqué le pré-test de compétences de production écrite sur les apprenants du groupe expérimental le $3 / 2 / 2018$. Puis elle a corrigé le test à la lumière du barème de notation préparé. 


\section{L'expérimentation}

Après avoir appliqué le pré- test, l'expérimentation a débuté le 4/2/2018 et s'est terminé le 15/3/2018. Elle a donc durée six semaines. C'est la chercheuse elle-même qui a dirigé le contenu du programme proposé aux apprenants de deuxième année secondaire.

\section{Application du post-test après}

l'enseignement du programme

proposé:

Après l'enseignement de l'unité proposée, la chercheuse a appliqué le post test aux apprenants du groupe expérimental le 16/3/2018 pour vérifier l'éfficacité du programme proposé basé sur l'approche actionnelle pour développer quelques compétences de la production écrite en FLE chez les apprenants de deuxième année secondaire. Puis la chercheuse a corrigé le test à la lumière du barème de notation préparé.

\section{Les résultats de la recherche}

Analyse statistique des résultats

obtenus:
Le pré/post test se divise en quatre questions, et il mesure quatre compétences principales qui comprennent un certain nombre de souscompétences. Chaque question mesure une compétence principale comme suit:

- La première question mesure la compétence (1) qui est de construire des phrases simples et complètes à l'aide de quelques mots.

- La deuxième question mesure la compétence (2) qui est de rédiger quelques phrases complètes et significatives à partir de certaines images données.

- La troisième question mesure la compétence (3) qui est de mettre les phrases en ordre chrolonogique pour formuler un texte complet en rapport avec le week-end.

- La quatrième question mesure la compétence (4) qui est de mettre les phrases en ordre chrolonogique pour formuler un texte complet en rapport avec les vacances.

- Les coefficients de corrélation entre les deux corrections pour le pré/post test de la production écrite d'un texte narrative. 
Tableau (1): les corrélations de Pearson entre les deux correctrices du pré/post test

\begin{tabular}{|c|c|c|c|c|c|}
\hline & $\begin{array}{l}\text { Pré test et Post } \\
\text { test }(\mathrm{c} 1 \text { et c2) }\end{array}$ & $\begin{array}{l}\text { Nombre des } \\
\text { apprenants }\end{array}$ & moyenne & $\begin{array}{c}\text { Ecart } \\
\text { type }\end{array}$ & $\begin{array}{c}\text { Corrélation de } \\
\text { Pearson }\end{array}$ \\
\hline \multirow{2}{*}{ Question 1} & $\mathrm{C} 1$ & 30 & 4.65 & 1.99 & \multirow{2}{*}{$0.620 * *$} \\
\hline & $\mathrm{C2}$ & 30 & 10.63 & 1.78 & \\
\hline \multirow{2}{*}{ Question 2} & C1 & 30 & 1.47 & 1.17 & \multirow{2}{*}{$0.442 * *$} \\
\hline & $\mathrm{C2}$ & 30 & 6.42 & 2.02 & \\
\hline \multirow{2}{*}{ Question 3} & C1 & 30 & 1.17 & 1.20 & \multirow{2}{*}{$0.482 * *$} \\
\hline & $\mathrm{C2}$ & 30 & 6.08 & 1.75 & \\
\hline \multirow{2}{*}{ Question 4} & C1 & 30 & 0.83 & 1.32 & \multirow{2}{*}{$0.516 * *$} \\
\hline & $\mathrm{C2}$ & 30 & 5.53 & 1.91 & \\
\hline
\end{tabular}

Significatif au niveau 0.01

Ce tableau affirme que les coefficients de corrélation entre les deux corrections sont significatifs au niveau statistique 0.01 . Ces résultats englobent le test total et les quatre questions. Les corrélations de Pearson sont: $(0.620,0.442,0.482,0.516)$.

\section{Analyse statistique concernant la première compétence:}

Il ya une différence statistique significative entre les moyennes des notes de l'échantillon de l'étude au pré-test et au post test en ce qui concerne la première compétence qui est la capacité des apprenants à construire des phrases simples et complètes à l'aide de quelques mots mesurée par la question (1), en faveur du post test.

Tableau (2): La valeur de $(T)$ pour la première compétence

\begin{tabular}{|c|c|c|c|c|c|c|}
\hline \hline Groupe & $\begin{array}{c}\text { Nombre } \\
\text { d'apprenants }\end{array}$ & Moyenne & Ecart type & $\begin{array}{c}\text { df (degré } \\
\text { de liberté) }\end{array}$ & $\begin{array}{c}\text { Valeur } \\
\text { de (T) }\end{array}$ & $\begin{array}{c}\text { Taille de } \\
\text { l'effet }\end{array}$ \\
\cline { 1 - 4 } pré test & 30 & 4.65 & 1.99 & 29 & \multirow{2}{*}{$12.23 * *$} & \multirow{2}{*}{0.84} \\
\hline post test & 30 & 10.63 & 1.78 & & \\
\hline \hline
\end{tabular}

Significatif au niveau 0.01

En observant le tableau ci-dessus, on peut remarquer que la valeur de $(\mathrm{T})$ est $12.23^{* *}$ en faveur du post test, et elle est significative au niveau 0.01. Cela affirme que l'unité proposée a contribué à améliorer et à développer cette compétence chez les apprenants de deuxième année secondaire.

\section{Analyse statistique concernant la}

\section{deuxième compétence:}

Il ya une différence statistique significative entre les moyennes des notes de l'échantillon de l'étude au pré-test et au post test en ce qui concerne la deuxième compétence qui est la capacité des apprenants à rédiger quelques phrases complètes et significatives àpartir de 
certaines images données mesurée par la question (2), en faveur du post test.

Tableau (3): La valeur de ( $\mathrm{T})$ pour la deuxième compétence

\begin{tabular}{|c|c|c|c|c|c|c|}
\hline \hline Groupe & $\begin{array}{c}\text { Nombre } \\
\text { d'apprenants }\end{array}$ & Moyenne & $\begin{array}{c}\text { Ecart } \\
\text { type }\end{array}$ & $\begin{array}{c}\text { df (degré de } \\
\text { liberté) }\end{array}$ & $\begin{array}{c}\text { Valeur } \\
\text { de (T) }\end{array}$ & $\begin{array}{c}\text { Taille de } \\
\text { l'effet }\end{array}$ \\
\cline { 1 - 3 } pré test & 30 & 1.47 & 1.17 & 29 & $10.56 * *$ & 0.79 \\
\cline { 1 - 3 } post test & 30 & 6.42 & 2.02 & 29 & \\
\hline \hline
\end{tabular}

Significatif au niveau 0.01

En regardant le tableau ci-dessus, la valeur de $(\mathrm{T})$ est $10.56^{* *}$ en faveur de post test, et elle est significative au niveau 0.01. Cela assure que l'unité proposée a aidé à développer cette compétence chez les apprenants de deuxième année secondaire.

\section{Analyse statistique concernant la}

\section{troisième compétence:}

Il ya une différence statistique significative entre les moyennes des notes de l'échantillon de l'étude au pré-test et au post test en ce qui concerne la troisième compétence qui est la capacité des apprenants à mettre les phrases en ordre chrolonogique pour formuler un texte complet en rapport avec le week-end mesurée par la question (3), en faveur du post test.

Tableau (4): La valeur de (T) pour la troisième compétence

\begin{tabular}{|c|c|c|c|c|c|c|}
\hline \hline Groupe & $\begin{array}{c}\text { Nombre } \\
\text { d'apprenants }\end{array}$ & Moyenne & $\begin{array}{c}\text { Ecart } \\
\text { type }\end{array}$ & $\begin{array}{c}\text { df (degré de } \\
\text { liberté) }\end{array}$ & $\begin{array}{c}\text { Valeur } \\
\text { de (T) }\end{array}$ & $\begin{array}{c}\text { Taille de } \\
\text { l'effet }\end{array}$ \\
\cline { 1 - 4 } pré test & 30 & 1.17 & 1.20 & \multirow{2}{*}{29} & \multirow{2}{*}{$13.83 * *$} & \multirow{2}{*}{0.87} \\
\cline { 1 - 3 } post test & 30 & 6.08 & 1.75 & & \\
\hline \hline
\end{tabular}

Significatif au niveau 0.01

D'après ce tableau ci-dessus, on peut noter que la valeur de $(\mathrm{T})$ est $13.83^{* *}$ en faveur de post test, et elle est significative au niveau 0.01. Cela nous montre que l'unité proposée a développé dans une large mesure cette compétence chez les apprenants de deuxième année secondaire.

\section{Analyse statistique concernant la} quatrième compétence:
Il ya une différence statistique significative entre les moyennes des notes de l'échantillon de l'étude au pré-test et au post test en ce qui concerne la quatrième compétence qui est la capacité des apprenants à mettre les phrases en ordre chrolonogique pour formuler un texte complet en rapport avec les vacances mesurée par la question (4), en faveur du post test. 
Tableau (5): La valeur de (T) pour la quatrième compétence

\begin{tabular}{|c|c|c|c|c|c|c|}
\hline \hline Groupe & $\begin{array}{c}\text { Nombre } \\
\text { d'apprenants }\end{array}$ & Moyenne & $\begin{array}{c}\text { Ecart } \\
\text { type }\end{array}$ & $\begin{array}{c}\text { DF (degré } \\
\text { de liberté) }\end{array}$ & $\begin{array}{c}\text { Valeur } \\
\text { de (T) }\end{array}$ & $\begin{array}{c}\text { Taille de } \\
\text { l'effet }\end{array}$ \\
\cline { 1 - 4 } pré test & 30 & 0.83 & 1.32 & \multirow{2}{*}{29} & \multirow{2}{*}{$10.75^{* *}$} & \multirow{2}{*}{0.80} \\
\cline { 1 - 3 } post test & 30 & 5.53 & 1.91 & & \\
\hline \hline
\end{tabular}

Significatif au niveau 0.01

Les résultats du tableau ci-dessus indique que la valeur de $(\mathrm{T})$ est $10.75^{* *}$ en faveur de post test, et elle est significative au niveau 0.01. Par conséquent, il ya un grand développement concernant cette compétence chez les apprenants de deuxième année secondaire grâce à l'unité proposée.

\section{Analyse statistique concernant les notes totales au pré/post test}

Il ya une différence statistique significative entre les moyennes des notes de l'échantillon de l'étude expérimentale au pré-test et au post test portant sur les compétences de la production écrite, en faveur de post test.

Tableau (6): Les valeurs de $(T)$ pour le pré/ post test

\begin{tabular}{|c|c|c|c|c|c|c|}
\hline \hline Groupe & $\begin{array}{c}\text { Nombre } \\
\text { d'apprenants }\end{array}$ & Moyenne & $\begin{array}{c}\text { Ecart } \\
\text { type }\end{array}$ & $\begin{array}{c}\text { df (degré de } \\
\text { liberté) }\end{array}$ & $\begin{array}{c}\text { Valeur } \\
\text { de (T) }\end{array}$ & $\begin{array}{c}\text { Taille de } \\
\text { l'effet }\end{array}$ \\
\cline { 1 - 4 } pré test & 30 & 8.12 & 4.00 & \multirow{2}{*}{29} & \multirow{2}{*}{$14.83 * *$} & \multirow{2}{*}{0.88} \\
\cline { 1 - 3 } post test & 30 & 28.66 & 6.03 & & \\
\hline \hline
\end{tabular}

Significatif au niveau 0.01

D'après ce tableau ci-dessus, on peut observer que la valeur de (T) est $14.83 * *$ en faveur de post test, et elle est significative au niveau 0.01. Cela affirme que l'unité proposée a aidé les apprenants de deuxième année secondaire à développer quelques compétences de la production écrite en FLE. En outre, ils ont progressé de manière remarquable.

\section{Discussion et interpretation}

D'après les résultats précédents, on peut remarquer $\mathrm{qu}^{\prime}$ il y a une différence significative entre les moyennes des notes des apprenants du groupe expérimental au pré-test et au post test concernant les compétences de la production écrite en faveur de post test, ce qui met evidence l'effet positif de l'unitée proposée.

On déduit donc que le programme basé sur l'approche actionnelle a eu une grande influence sur le développement de quelques 
compétences de la production écrite chez les apprenants de deuxième année secondaire.

Les deux tableaux $(2,3)$ représentent les résultats obtenus en ce qui concerne les réponses aux questions $(1,2)$ qui ont visé à mesurer la capacité des apprenants à construire des phrases simples, significatives, et complètes à l'aide de quelques mots ou de quelques images. Cela comprend la bonne structure de la phrase, la ponctuation, la conjuguaison correcte de verbes, la formulation des phrases logique, la transcription compréhensible, l'utilisation de déterminants, le choix du vocabulaire précis, le choix de temps convenable, toutes sortes d'accord, les accents, le respect de la majuscule et de la minuscule et l'orthographe correcte des mots. La lecture de ces tableaux nous indique une amélioration considérable.

En effet, les apprenants éprouvaient de plusieurs difficultés au niveau de ces compétences. C'est parce qu'ils ne s'entrainaient pas suffisamment aux activités en rapport avec celles-ci.

Les activités proposées au programme ont aidé à rémedier aux difficultés rencontrées. En plus, le fait qu'ils ont utilisé la grille de co-évaluation tout au lang de l'exécution des travaux écrits les a rendus plus prudents.

Les deux tableaux $(4,5)$ représentent les résultats obtenus concernant les réponses aux questions $(3,4)$ qui ont visé à mesurer la même compétence chez les apprenants. Cette compétence se représente à leur capacité à mettre les phrases en ordre chronologique pour formuler des textes complets et compréhensibles. Cela à son tour englobe la génération d'idées diverses lièes aux sujets demandés, le choix du vocabulaire précis, la variété du vocabulaire, l'utilisation de connecteurs, les éléments de répétition, la pronominalisation, la ponctuation, la conjuguaison des verbes, le choix de temps convenable, l'ordre logique d'évènements et la cohérence textuelle.

En analysant ces deux tableaux, nous montrent une grande amélioration. Cela veut dire on peut déduire que les apprenants ont marqué une grande progression lors du post test.

En effet, la majorité des apprenants dans le pré-test ne connaissaient pas comment structurer des textes.

En outre, au début du semestre, la chercheuse a observé en discutant avec les apprenants que les outils nécessaires pour formuler et organiser les idées en rédigant des textes complèts leur manquaient totalement.

Pour atteindre à ce résultat, le programme proposé a vraiment présenté des connaissances et des activités utiles pour 
consolider, enrichir, et améliorer les compétences précédemment mentionnées.

Lors du pré-test et au début de l'intervention du programme proposé, la chercheuse a observé les points suivants:

- L'incapacité des apprenants à choisir le vocabulaire approprié.

- L'incapacité des apprenants à varier le vocabulaire utilisé, par conséquent ils n'ont pas pu s'exprimer.

- L'incapacité des apprenants à formuler des phrases complètes, correctes, et significatives.

- L'incapacité des apprenants à produire des idées diverses et non répétés.

- L'incapacité des apprenants à traduire écritement leurs idées.

- L'incapacité des apprenants à organiser les idées en paragraphes.

- L'incapacité des apprenants à conjuguer correctement les verbes au passé composé, et à l'imparfait. En outre, ils ont recouru à faire un mélange entre le présent et le passé.

- L'incapacité des apprenants à utiliser les connecteurs.

- L'incapacité des apprenants à rédiger ou transcrire de manière compréhensible, organisée, et facile à lire.

- L'incapacité des apprenants à utiliser la ponctuation correcte et compatible.
- L'incapacité des apprenants à rédiger des évènements passés en ordre chrolonogique.

- L'incapacité des apprenants à interagir avec les camarades, parce qu'ils ne s'entrainaient pas à agir ensemble.

- La perte du temps consacré pour accomplir les travaux écrits demandés.

Mais à la fin de l'étude expérimentale, la chercheuse a observé un grand perfectionnement chez les apprenants. Tout au long du déroulement de l'intervention, la chercheuse remarquait un développement dans la performance de la plupart des apprenants dans la production écrite.

On peut dire que le programme proposé basé sur 1'approche actionnelle était éfficace pour développer les compétences de la production écrite chez les apprenants de la deuxième année secondaire.

\section{Cela met en évidence que le} programme proposé a permis de:

1- Considérer les apprenants comme des acteurs sociaux qui peuvent participer librement dans différentes situations d'interaction dans la vie quotidienne. Cela conduit à pratiquer et maîtriser la langue cible. 
2- Mettre les apprenants en situation d'interactivité mutuelle pendant une longue période.

3- Soutenir les principes de participation positivement, et de motivation chez les apprenants.

4- Aider les apprenants à travailler en équipe, c'est-à-dire ce programme renforce l'ésprit d'équipe.

5- Aider les apprenants à acquérir de nouvelles connaissances et de nouvelles compétences.

6- Aider les apprenants à échanger les informations et les points de vue, à les corriger, à les améloirer, et à les réutiliser.

7- Aider les apprenants à rédiger librement, c'est la meilleure façon de s'exprimer, et d'apprendre de nouveau vocabulaire en langue cible.

8- Rendre les apprenants très actifs en classe à travers de l'interaction avec les collègues et de la réalisation des tâches actionnelles écrites qui leur ont été confiées en pratiquant la langue cible dans une production concrète. C'est très différent de ce qu'on a l'habitude de faire.

9- Donner aux apprenants l'opportunité de distinguer entre la cause, et la conséquence, et retrouver l'ordre logique en leurs écrits.

10-Rendre la classe comme éspace sociale dans laquelle les apprenants vivent naturellement un certain nombre de tâches authentiques et significatives.

11-Inciter les apprenants à entrer de manière active dans la langue française.

En outre, le programme proposé était éfficace grâce aux points positifs suivants:

- Les activités variées présentées tout au long du déroulement de l'unité proposée ont bien contribué au développement de la qualité de produits écrits des apprenants.

- Les textes à lire proposés aux leçons de l'unité expérimentale ont permis aux apprenants d'enrichir leur vocabulaire, de varier leurs idées, et de respecter la structure de textes.

- Les questions proposées dans chaque leçon ont offert la chance de pratiquer plusieurs types d'activités.

- Les textes à rédiger présentés ont soutenu les apprenants à faire des progrès dans leurs travaux écrits.

- La grille de co-évaluation appliquée lors de la rédaction de textes a développé la qualité de leurs produits écrits.

En résume, cette unité proposée à la lueur de l'approche actionnelle a recouru à créer des 
situations et des tâches concrètes afin d'activer l'apprentissage et en vue de développer des compétences langagières chez les apprenants en leur permettant d'interagir facilement dans la vie sociale.

En plus, on peut dire que l'approche actionnelle aide les apprenants à dévenir des futurs citoyens susceptibles d'étudier, de travailler, de s'impliquer, et de vivre à l'étranger.

\section{Recommandations}

À la lumière de la discussion des résultats obtenus en regard du cadre théorique de cette recherche, on peut présenter les recommandations suivantes:

- Il est profitable et indispensable d'organiser des stages pour entraîner les enseignants à la bonne utilisation de programmes basés sur l'approche actionnelle dans le processus de l'enseignement- apprentissage.

- Les apprenants doivent être encouragés et incités à acquérir les méthodes qui leur permettent d'avoir envie d'écrire en français.

- Les enseignants doivent adopter de nouvelles approches qui porte sur le respect de l'autonomie de l'apprenant et de sa responsabilité tout au long de son apprentissage.
- L'enseignant de classe doit rémedier aux difficultés lièes à la timidité , à la peur de la faute, et au refus de se impliquer lors de la pratique de différentes activités écrites à travers de donner aux apprenants la liberté pour écrire beaucoup.

\section{Suggestions de la recherche}

À la fin de cette recherche, la chercheuse propose quelques recherches qui peuvent-être traité dans d'autres études:

- Elaborer un programme basé sur l'approche actionnelle pour développer les compétences de l'expression orale chez les étudiants du cycle secondaire.

- Étudier l'éfficacité d'un programme basé sur l'approche actionnelle pour développer les compétences de la lecture chez les étudiants du cycle secondaire.

- Étudier l'efficacité de l'approche interactionnelle et Web social sur le développement de la production écrite chez les étudiants du cycle secondaire.

- Elaborer un programme basé sur le scénario pédagogique pour développer les compétences de la compréhension écrite chez les étudiants du cycle secondaire.

- Mesurer l'effet de l'approche communico- actionnelle sur l'oral ou l'écrit dans des différents cycles éducatifs. 
- Piloter des programmes pour entraîner les enseignants dans différents cycles éducatifs à l'utilisation de l'approche actionnelle.

\section{Les références}

1) Abbas, M. (1991). "les erreurs fréquentes dans 1'emploi des temps des verbes dans 1'expression écrite des élèves de la première année secondaire du français , comme deuxième langue étrangère". Thèse de Maîtrise non publiée .Faculté de Jeunes Filles. Université d'AinChams.

2) Abbas, M. (2002). "Stratégies proposées pour l'enseignement du texte littéraire au Cycle secondaire", Thèse de Doctorat, Faculté de Jeunes- Filles, Université d'Ain- Chams.

3) Abd Rabou, D. ( 2008). " Efficacité d'un programme proposé basé sur l'auto apprentissage pour le développement des compétences de la lecture, de l'écriture et de l'autonomie chez les futurs enseignants à la section de français à la faculté de pédagogie ". Thèse de doctorat. Université $\mathrm{D}^{\prime}$ Ain Chams.

4) Abdel Fattah, A. (1997)." Les erreurs fréquentes dans l'emploi des prépositions dans l'expression écrite des élèves de la deuxième année secondaire (Français deuxième langue)". Thèse de Doctorat non- publiée. Faculté de Jeunes Filles. Université d'Ain Chams.

5) Abdelli, k. (2011)." Stratégies mises en oeuvre à l'apprentissage du FLE en Algérie: Cas d'une production écrite d'un texte narratif en 2ème année secondaire", Thèse de Magistère. Faculté des lettres et des langues, École Doctorale de français, Université Abou Bakr Belkaïd.

6) Abd-Rabou, A. (2003). "Développement des compétences de l'expression orale en français, deuxième langue étrangère chez les étudiants du Cycle secondaire", Thèse de Magistère, Faculté de Pédagogie, Université Ain- Chams.

7) Agami, Ch. (2010)." Efficacité d'un programme proposé basé sur l'approche $\mathrm{du}$ processus de l'écriture pour le développement de quelques compétences de la production écrite et les attitudes des élèves du cycle secondaire". Thèse de Doctorat (non publiée). Faculté de Jeunes Filles. Université d'Ain - Chams.

8) Barrié, M. (2012). L'approche actionnelle: La réalité pratique et ses limites d'application à l'école primaire. Ecole Interne IUFM Midi-Pyrénées/UT2 .En partenariat avec: UT1, UT3 et CU-JF 
Champollion .Mémoire de master 2. Master EFE-ESE

9) Bazelaire, E. (2012). Jeu et perspective actionnelle dans l'enseignement des langues vivantes au primaire. Université d'Orléans et de tours. Disponible sur: http://dumas.ccsd.cnrs.fr/docs/00/76/53/0 1/PDF/MA_moire_Soutenance_Bazelair e_Final_Et_Annexes_1_.pdf Consulté le 15 Mars 2013.

10) Bourguignon, C. (2007). Apprendre et enseigner les langues dans la perspective actionnelle : Le scénario d'apprentissage-action; Conférence à l'assemblée Générale de l'APLV à Grenoble, par Claire Bourguignon, Maître de conférences HDR, IUFM de l'Académie de Rouen claire.bourguignon@rouen.iufm.fr

11) Bourguignon, C. (2010). L'apprentissage des langues par l'action. In L'approche actionnelle dans l'enseignement des langues. Pp. 49-77.

12) Catroux, M. (2006). Perspective coactionnelle et TICE: quelles convergences pour $1^{\prime}$ enseignement de la langue de spécialité?. Communication donnée dans le cadre des journées d'Etude de 1'EA2025. Disponible sur: http://www.langues-vivantes.u-
bordeaux2.fr/frsa/pdf/Catroux.pdf

Consulté le 20 Mars 2013.

13) Charline, R. (2010). "L'enfant sourd et la production écrite de texts narratifs :pistes d'évaluation", Mémoire de Master 1, Recherche Sciences du langage, Université Stendhal ,Dumas-00520135, Version 1 - 22.

14) Conseil de l'Europe. (2001). Conseil de la coopération culturelle, Comité de l'Éducation, Division des langues vivantes : Cadre européen commun de référence pour les langues : apprendre, enseigner, évaluer. Paris: Ed. Didier.

15) Cornaire, C et Raymond, P. M (1999) : La production écrite. Clé International. Collection: Didactique des langues étrangères. Paris.

16) Coste, D. (2009). « Tâche, progression, curriculum ». In É. Rosen (dir). La perspective actionnelle et l'approche par les tâches en classe de langue, numéro spécial de la revue Le français dans le monde, recherches et applications, $\mathrm{n}^{\circ} 45$, pp.15-24.

17) Eid, M. (2006). "L'effet de l'utilisation d'un programme basé sur les modes des histoires sur le développement de l'expression écrite en françis chez les étudiants de la première année à la faculté de pédagogie de l'université de 
Minia". Thèse de Magistère non publiée . Faculté de pédagogie. Université de Minia.

18) El Ahwal, W. (1991). "Développement de certaines capacités d'expression écrite en langue française chez les étudiants des facultés de pédagogie". Thèse de Doctorat non publiée. Faculté de pédagogie de Damiette. Université de Mansoura.

19) El Battrawy, A.E. (1997) . " les erreurs fréquentes dans l'emploi des prépositions dans 1'expression écrite des élèves de la deuxième année secondaire (Français deuxième langue)" . Thèse de Maîtrise non publiée. Faculté de jeunes filles . Université d'Ain Chams.

20) El Kassas, B. (2002). "Programme proposé en vue de développer les stratégies d'apprentissage à écrire en FLE chez les futurs -enseignants en Egypte". Thèse de Doctorat non- publiée. Faculté de pédagogie. Université d'AinChams.

21) Elise, P. (2012). L' aboutissement de 1' approche actionnelle. Disponible sur: http://dumas.ccsd.cnrs.fr/docs/00/73/57/9 7/PDF/pannequin_elise.pdf Consulté le 27 Février 2013.

22)El-kouche, A. (2004)." l'efficacité de l'emploi de l'approche par le processus sur le développement des habilités d'écriture et les attitudes des étudiants du département de langue française". Thèse de Doctorat (non publiée). Université de Caen Basse-Normandie.

23) El Naghy, A. (2016). "Programme proposé fondé sur l'utilisation des cyberquêtes pour développer quelques compétences de l'expression écrite en langue française chez les élèves du cycle secondaire". Thèse de Doctorat. Faculté de pédagogie. Université de Port-Saïd.

24)Farouk, H. (2004). "L'efficacité de l'apprentissage coopératif et de l'enseignement individualisé sur le développement de 1'aspect grammatical en expression écrite chez les étudiants des facultés de pédagogie". Thèse de Doctorat non publiée. Faculté de pédagogie. Université de Zagazig.

25) Gad, M. (1990). "les fautes grammaticales communes dans les écrits des étudiants de la deuxième année secondaire en français comme deuxième langue étrangère, étude d'évaluation ". Thèse de Maîtrise non-publiée. Faculté de pédagogie. Université de Mansourah.

26) Gomes, L. (2012). La perspective actionnelle: la création d'un blog1 en classe de FLE comme exemple d'un projet pédagogique actionnel. 
(Universidade Federal da Paraíba).

Revista Letr@Viv@v.11,n.1/UFPB-

DLEM/ISSN 1517-3100 / p. 39 - 45

27) Gonzalez, R. (2012). Perspective actionnelle et autonomie chez l'apprenant. Université Stendhal. Master 1-Mention Sciences du langage. Dossier : Courants méthodologiques et analyse de méthodes (Avant évolution des courants méthodologiques).

28)Hafez, H. (2001)." Efficacité de l'apprentissage cooperative sur l'acquisition des compétences de la performance écrite en français (deuxième langue étrangère) chez les étudiants du cycle secondaire ". Revue de la lecture et de la connaissance. Faculté de pédagogie. Université d'Ain Chams.

29)Hamed, M. (2004)." Efficacité de la stratégie de l'apprentissage cooperative en vue de déelopper les compétences de $1^{\prime}$ expression écrite en langue française chez les élèves du cycle préparatoire aux écoles expérimentales", Thèse de Magistère (non publiée). Faculté de pédagogie. Université de Hélouân.

30) Ibrahim, H. (1995)." Effet d'un programme proposé en vue de développer les compétences de la performance écrite en français deuxième langue étrangère chez les élèves du cycle secondaire". Thèse de Maîtrise non publiée. Faculté de pédagogie. Université de Tanta.

31) Ibrahim, M. (1995). "Programme proposé eu vue de développer le vocabulaire $\mathrm{du}$ français chez les étudiants au cycle secondaire". Thèse de Doctorat non - publiée. Faculté de Jeunes Filles. Université d'Ain - Chams.

32) Khalifa, M. (2009). "Un modèle constructiviste pour apprendre la grammaire de français et son efficacité à développer les compétences de l'expression écrite chez les élèves du cycle secondaire". Thèse de Magistère. Faculté de pédagogie. Université de Hélouân.

33) Mahmoud, D. (2005)." Efficacité de l'approche naturelle sur le développement de l'expression écrite en langue française chez les étudiants du cycle préparatoire". Thèse de Magistère non publiée. Faculté de pédagogie. Université de Hélouân.

34) Mohammed, Ch. (2003)." L'efficacité d'une stratégie proposée établie sur le style de l'exercice guidé et la résolution des problèmes dans le développement de quelques compétences de l'expression écrite libre chez les étudiants de 
quatrième année de section de français à la faculté de pédagogie de Tante". Thèse de Magistère non publiée . Faculté de pédagogie. Université de Tanta.

35) Mohammed, N. (2005). "Efficacité de 1' approche constructiviste sur le développement de quelques compétences de l'expression écrite fonctionnelle chez les étudiants-maîtres du département de français".Thèse de Magistère (non publiée). Faculté de Jeunes Filles. Université d'Ain - Chams.

36) Mohammed, N. (2017). "Effet de l'approche constructivisite pour développer quelques compétences de la production écrite chez les étudiants du cycle secondaire". Faculté de pédagogie.

37) Nissen, E. (2003). Apprendre une langue en ligne dans une perspective actionnelle. Effets de l'interaction sociale. Thèse de Doctorat. Université Strasbourg I - Louis Pasteur.

38) Nissen, E. (2004) . Importance du scénario pédagogique dans l'apprentissage d'une langue étrangère en ligne. P. 14-24

39) Orvoën, C. (2012). Proposition de sequences didactiques actionnelles: Comment articuler l'étude formelle de la langue étrangère et son usage en interaction écrite et orale? version 1 .
UFR LLASIC - Langage, Lettres et Arts $\mathrm{du}$ Spectacle, Information et Communication. Département des Sciences du Langage et du Français Langue Étrangère. Section de Didactique $\mathrm{du}$ Français Langue Étrangère. Disponible sur: http: // Dumas.ccsd.cnrs.fr/ docs/00/75/18/91/ pdf/ MA-moire-M2-Orvoa-n-cindy.pdf Consulté le 25 Janvier 2013.

40) Pérès, L. (2013). L'évolution des pratiques dans l'enseignement des langues vivantes étrangères à l'école primaire est-elle en phase avec l'évolution des approches de cet enseignement ?. Mémoire Demaster 2 Master EFE-ESE. École Interne IUFM Midi-Pyrénées/UT2. En partenariat avec: UT1, UT3 et CU-JF Champollion.

41) Peris, M. (2009). “L'éducation pour l'autonomie: un nouveau modèle d'enseignement", p. 110 in AAEL.

42) Plane, S. (2004). L'écriture et son apprentissage à l'école élémentaire, Coll. Repères, Ed. Dépôt Légal, la France.

43) Pluskwa, D\& Willis, D \& Willis, J. (2009). L'approche actionnelle en pratique: la tâche d'abord, la grammaire ensuite ! Dans « L'approche actionnelle dans l'enseignement des langues. Onze articles pour mieux comprendre et faire 
le point». Paris: Ed. Maison des Langues.

44) Puren, C. (2013). «De l'approche communicative à la perspective actionnelle, ou de l'interaction à la coaction ». Résumé d'une conférence au XIIIe colloque de l'Association des Enseignants de Japonais de France, Rennes. En ligne http://www.christianpuren.com/monagenda/rennes-aejf/

45) Puren, Ch. (2006). La perspective actionnelle. Vers une nouvelle coherence didactique. Le Français dans le monde. http://www.rpkansai.com/bulletins/pdf/0 24/073 087 Puren.pdfConsulté le 10 $\underline{\text { Septembre2013. }}$.

46) Puren, Ch. (2009). Variations sur la perspective de l'agir social en didactique des langues-cultures: vers une perspective co-actionnelle-co-culturelle, dans Recherches et Applications, LFDM $\mathrm{n}^{\circ} 45$, La perspective actionnelle et l'approche par les tâches en classe de langue. Paris: Clé International.

47) Puren, Ch. (2010). Les manuels récentsde français langue étrangère: entre perspective actionnelle et approche communicative. Université de SaintÉtienne. Disponible sur:
/volume4i2/papers/PurenFULGOR2010. pdfConsulté le 16 Juillet 2013.

48) Puren, Ch. (2011). Mise en oeuvre de la perspective actionnelle: analyse comparative de la «tâche finale » dans deux manuels de FLE, Latitudes 2 (2009) \& version originale 1 (2009)http://www.christianpuren.com/m es-travaux-liste-et-liens/2011d/Mars $\underline{2011}$

49) Pütz, W. (2009). L'usage de la méthode tâche actionnelle au cours du FLE selon l'exemple $\mathrm{du}$ poème. Ma bohème d'Arthur Rimbaud, Analyse du stage en situation: Outils de didactique et enseignement du FLE. Université de Cologne Séminaire roman.

50) Ranst, L. (2006). Wiki et perspective actionnelle : Analyse pédagogique d'un projet européen. Disponible sur: http://www.cesep.be/Etudes/Techno/Wik i.pdf Consulté le 23 Février 2013.

51) Robert, J. P. (2002). Dictionnaire pratique de didactique du FLE, Paris, Ophrys

52) Rosen, É. (2009). «La perspective actionnelle et l'approche par les tâches en classe de langue ». Le Français dans le Monde. "Recherches et applications" $\mathrm{n}^{\circ}$ 45. Paris : CLE international-FIPF.

http://ehlt.flinders.edu.au/deptlang/fulgor 
53) Sadek, W.M. (2005)."Utilisation du portfolio pour développer les compétences de l'écriture chez les étudiants du département de français des facultés de pédagogie". Thèse de magistère .Université de Mansoura.

54) Saleh, M. (1994)." Les activités langagières et leur effet sur le développement de quelques compétences écrites chez les élèves de la première année secondaire". Thèse de Magistère non- publiée. Faculté de péagogie.Université d' Ain - Chams.

55) Saleh, N. (2015). "Efficacité de l'utilisation de l'approche actionnelle pour développer quelques compétences de la compréhension écrite chez les étudiants à la faculté de pédagogie". Thèse de Doctorat. Faculté de pédagogie d'Al Arish. Université de Canal de Suez.

56) Salem, A. (1992). "Effet de l'utilisation des méthodes communicatives sur les niveaux oral et écrit du français comme deuxième langue étrangère chez les élèves de la première année secondaire". Thèse de Magistère non publiée. Faculté de pédagogie. Université de Zagazig.

57) Sara, Kh. (2011). "Pour une compétence de la production écrite chez les apprenants de la quatrième année moyenne", École Doctorale, Antenne de l'université Kasdi Merbah, Algérie.

58) Sarah, B. (2008). En quoi la mise en oeuvre d'une approche actionnelle des langues favorise-t-elle les apprentissages?. Institut Universitaire de formation des maîtres. Académie de Montpellier. Ecole de VentenacCabardès. Classe de CM1-CM2

59) Shaïri, H. (2009). Le rôle de la tâche dans l'augmentation de la motivation des apprenants du FLE. Université Tarbiat Modarres. Pazhuhesh-e Zabanha-ye Khareji, No. 53, Special Issue, French, pp. $43-57$

60) Sinh, Ph. (2006). "Étude des erreurs en production écrite des étudiants en français à l'école normale supérieure de Thàinguyên", Mémoire de fin d'études post-universitaires, École supérieure de langues étrangères, Université nationale de Hanoi

61) Springer, C. (2009). « La dimension sociale dans le CECR : pistes pour scénariser, évaluer et valoriser l'apprentissage collaboratif ». In É. Rosen (dir). La perspective actionnelle et l'approche par les tâches en classe de langue, numéro spécial de la revue Le français dans le monde, recherches et 
applications, $\mathrm{n}^{\circ} 45$, pp. 25-34. Paris : Clé International.

62) Tano, M. (2010). «Autonomie et guidage dans l'enseignement-apprentissage de l'espagnol de spécialité», VIII Rencontres Internationales du GERES / Bordeaux. Ecole Nationale d'Ingénieurs de Metz / Editions Maison des Langues.

63) Tardieu, C. (2006). Se former pour enseigner les langues à l'école primaire. Le cas de l'anglais. Ouvrage collectif sous la direction de Claire Tardieu. Ellipses. Paris. 269 pages.

64) Toruç, D. (2013). Comment peut-on faire développer l'autonomie des étudiants en classe de langue étrangères surtout en classe de FLE?; Turkish Studies - International Periodical For The Languages, Literature and History of Turkish or Turkic Volume 8/10, p. 659-669, Ankara-Turkey.

65) Trim, J.LM. (1997). Les Langues vivantes: apprendre, enseigner, êvaluer. Un cadre Européen Commun de Référence. Guide général d'utilisation. Strasbourg: Conseil de l'Europe, 41 p., multigr.
66) Zekri， I. (2003). "Efficacité d'un programme proposé en employant l'approche coopérative en vue de développer quelques compétences écrites de la dissertation, chez les étudiants de la faculté de pédagogie de Fayoum, section de français". Thèse de Doctorat non publiée. Faculté de pédagogie de Fayoum. Université de Caire. 